\title{
INTEGRATING BIOREACTOR SYSTEM TO SPEED UP TUMOUR INFILTRATING LYMPHOCYTES (TILS) IMMUNOTHERAPY COMMERCIALIZATION
}

\author{
A. D. BUDIYATI ${ }^{1,2}$, J. A. PAWITAN $3,4,5^{*}$ \\ 1Doctoral Program for Biomedical Sciences, Faculty of Medicine, Universitas Indonesia, Jakarta, Indonesia, ${ }^{2}$ Stem Cell and Cancer \\ Institute, Jakarta, Indonesia, ${ }^{3}$ Department of Histology, Faculty of Medicine, Universitas Indonesia, Jakarta, Indonesia, ${ }^{4}$ Stem Cell Medical \\ Technology Integrated Service Unit, Dr. Cipto Mangunkusumo General Hospital/Faculty of Medicine Universitas Indonesia, Indonesia, \\ ${ }^{5}$ Stem Cell and Tissue Engineering Research Center, Indonesia Medical Education and Research Institute (IMERI), Faculty of Medicine \\ Universitas Indonesia, Indonesia \\ Email: jeanneadiwip@gmail.com
}

Received: 23 Feb 2020, Revised and Accepted: 12 May 2020

\begin{abstract}
One of the major breakthroughs on adoptive cell transfer (ACT) is autologous T cells transfer that either derived from tumour infiltrating lymphocytes (TILs) or genetically engineered T cells, which express tumour recognizing receptors. Between those two, when the purpose is to treat solid tumours, a more impressive clinical trial result is demonstrated by TIL therapy. The efficacy of TIL therapy is due to its highly personalized approach in generating T cells from the patient's own tumour tissue. Regardless to its efficacy, TIL therapy in a commercial setting is limited, mainly due to the complexity and costs of cell expansion protocols. This issue has triggered the need to develop effective methods to expand TILs ex vivo, in a safe, efficient, and reproducible manner. In line with this need, there are two main types of bioreactors that have recently been used to generate $\mathrm{T}$ cells for ACT, which might suit the requirements for TIL manufacture. Therefore, in this review, we highlighted recent updates on TILs expansion protocol, particularly in autologous settings, as well as the aspect of each bioreactor to be considered before integrating them into TIL manufacturing process.
\end{abstract}

Keywords: Adoptive cell transfer, Immunotherapy, Tumour infiltrating lymphocytes, Bioreactor, Solid tumour

(C) 2020 The Authors. Published by Innovare Academic Sciences Pvt Ltd. This is an open access article under the CC BY license (http://creativecommons.org/licenses/by/4.0/) DOI: http://dx.doi.org/10.22159/ijap.2020v12i4.37244. Journal homepage: https://innovareacademics.in/journals/index.php/ijap

\section{INTRODUCTION}

Adoptive cell transfer (ACT) is a treatment that uses immune system cells to eliminate cancers. In cancer therapy, an ACT-based strategy exploits the killing potency of specific $\mathrm{T}$ cells to generate a robust immune-mediated anti-tumor response. This anti-tumor response is achieved by manipulating T cells ex vivo, away from the patient's immune-suppressive environment, followed by expansion and reinfusion back to the patient $[1,2]$.

Based on the way that T cells are manipulated, ACT-based strategy can be divided into two platforms, i.e. isolation of naturally occurring tumor-specific T cells from existing tumour masses, which is called tumour infiltrating lymphocytes (TILs) platform [1, 2], and genetic modification of blood-derived $\mathrm{T}$ cells to allow specific recognition of tumour cells, which is called chimeric antigen receptor (CAR) T-cell or T-cell receptor (TCR) platform [1-4]. Both platforms have demonstrated impressive, durable response in clinical settings to treat certain tumours, so that FDA approved CAR T-cell therapy for treating hematological malignancies and TIL therapy for treating melanoma and persistent cervical cancer $[1,2]$.

Despite various successes in treating B-cell leukaemia and lymphomas, CAR T-cell therapies have not demonstrated their efficacy in solid tumours. As hematological malignancies make up only a small fraction of all cancers, TIL platform is most likely to have broader applicability for treating solid tumours [2, 5, 6]. However, in autologous cell therapy, current TIL expansion protocols remain facing challenges that are mainly due to limited $\mathrm{T}$ cell number, which can be isolated from a patient tumour. Moreover, although a TIL' rapid expansion protocol has been developed decades ago by Rosenberg in 1988 [7], the protocol still needs to be simplified to allow efficient scale-up for TIL manufacturing. For this review, we searched various databases, i.e. Pubmed, Science Direct, Springer, and Innovare Academic Sciences, using keywords: "tumour infiltrating lymphocyte" and "Bioreactor" at 30 December 2019, without time limits. Only English literatures were used.

The main aim of this review is to present a brief outline of the basic concepts of TILs in treating solid tumours, as well as general protocols for ex vivo TIL expansion that are currently being used in clinical application. In addition, three bioreactors that have been used in many commercial ACT services, i.e. GE Wave, G-Rex, and Quantum are also discussed here along with general considerations of integrating them into TIL ex vivo expansion protocol.

\section{TIL basic concept in treating solid tumours}

TILs are a heterogeneous population of lymphocytes, which primarily consist of $\mathrm{T}$ and natural killer (NK) cells that naturally migrate into tumours and are potentially present in any solid tumour $[1,7,8]$. Among these two subsets, a more effective killing capability is owned by T-cells because they can recognize tumour associated antigens (TAAs) due to the presence of endogenous $\mathrm{T}$ cell receptors (TCRs). Therefore, exploiting TIL' killing capacity was studied extensively to target solid tumour, which further led to FDA approval for treating cervical cancer and metastatic melanoma [2, 5].

Compared to CAR T-cell platform in terms of treating solid tumours, TILs have several advantages. Despite both CAR T-cells and TILs have the ability to recognize TAAs, TILs have a wider coverage of TAAs. TILs that are extracted from tumour resection consist of clones that are able to recognize multiple TAAs, while CAR T-cells, which require an artificial engineered antigen receptor, can only recognize a single TAA. Moreover, since TILs are naturally formed to target tumour neoantigens, this platform offers minimum adverse effects that are caused by unpredicted off-target effects on other tissues. Table 1 describes the competitive advantages of TIL therapy on treating solid tumours in comparison with CAR T-cell platform [9].

Currently, TILs are implemented as autologous-based cell therapy, but generating highly activated $\mathrm{T}$ cells in sufficient numbers from tumours requires a protocol that is able to overcome the limited number of TILs, which are extracted from patient tumour tissue. Therefore, an effective and efficient expansion protocol is highly needed.

\section{General protocols for TIL ex vivo expansion}

Ex vivo expansion of TILs requires several steps to get a sufficient number of activated T cells. A preliminary report by Rosenberg et al. in 1988 paved the way for the development of a standard method for large-scale in vitro expansion of TILs that were isolated from a 
patient's tumour [7]. As depicted in fig. 1, the method involves three main steps. It starts with TIL extraction from a patient's tumour tissue, rapid expansion protocol (REP), which consists of TIL expansion using high dose IL-2 and co-culture with feeder cells to generate enough cells for ACT, and finally harvest and preparation of TILs before reinfusion back to the patient $[7,8,10,11]$. The following sections described those three steps that are involved in TIL ex vivo expansion.

Table 1: The competitive advantages of TIL therapy on treating solid tumors compared to CAR T-cell platform

\begin{tabular}{llll}
\hline References & Comparison & TILs & CAR T cells \\
\hline$[9]$ & Technology platform & No genetic modification & Genetic modification \\
{$[9]$} & T cells' source & Tumour tissue & Peripheral blood \\
{$[9]$} & Usage & Autologous, no HLA restriction & Autologous, no HLA restriction \\
{$[9]$} & Target coverage & Multiple tumour antigens & Single surface tumour antigen \\
{$[9]$} & Risk of adverse effect & Minimal chance of off-target effect on other & Potential off-target effect on other tissues \\
& & tissues & (immunogenic), cytokine release syndrome \\
{$[9]$} & Type of treatment & One time treatment & One time treatment \\
{$[9]$} & Utility & Solid tumours: evidence in melanoma and & Leukemias and lymphomas, no evidence in \\
& & cervical cancers & solid tumours \\
\hline
\end{tabular}

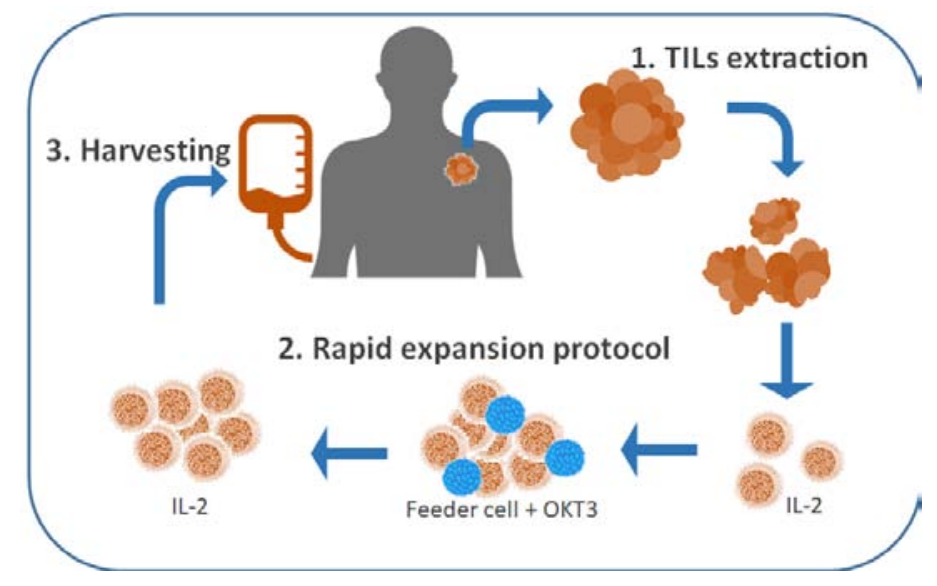

Fig. 1: TIL ex vivo expansion workflow $[7,8,10,11]$

There are three main steps in ex vivo TIl expansion: 1 . Extraction of TILs from a patient own tumour, 2. Rapid expansion protocol using high dose of IL-2 followed by activation by OKT3 expressing feeder cells, and expansion to billion numbers of TILs ex vivo, 3. Harvesting and preparation of ready to use expanded TILs in infusion bag to be re-infused back to a lymphocyte-depleted patient $[7,8,10,11]$

\section{TIL extraction}

In general, based on a previously described method by Dudley et al. in 2003 [8], there are three feasible methods for initiating TIL culture that started from the patient's dissected tumours. The first method is to grow TILs from multiple tumour fragments individually as explant cultures in IL-2 prior to culture expansion. The second method is by enzymatic digestion, and the third one is by using mechanical intervention to yield single cells and/or small aggregates from the tumour. The three methods were started from biopsy specimens that are free from normal and necrotic tissues. Biopsy specimens were then cut into 1 to $2 \mathrm{~mm}$ dimension using a sharp scalpel [8].

In the first method, each tumour fragments are placed in single wells of 24-well tissue culture plates with $2 \mathrm{ml}$ of complete medium (CM) and cultured. The CM contains $25 \mathrm{mmol} / \mathrm{l}$ HEPES $\mathrm{pH} 7.2$, penicillin and streptomycin (100 $\mathrm{U}$ and $100 \mu \mathrm{g} / \mathrm{ml}), 2 \mathrm{mmol} / \mathrm{l} \mathrm{L}$-glutamine, 5.5 x $10^{-5} \mathrm{~mol} / \mathrm{l} \beta$-mercaptoethanol, $10 \%$ human serum and $6000 \mathrm{IU} / \mathrm{ml}$ IL-2 in RPMI 1640. The culture is incubated in a humidified incubator at $37{ }^{\circ} \mathrm{C}$ with $5 \% \mathrm{CO} 2$, and daily inverted-microscope inspection is done to observe lymphocyte proliferation. This method takes about 1 to $2 \mathrm{w}$ to yield a monolayer of lymphocytes prior to further expansion [8].

In the second method, which uses enzymatic digestion, the tumour fragments are immersed in a medium that contains collagenase, hyaluronidase and DNAse in RPMI 1640 overnight with gentle agitation to cause enzymatic degradation. Following filtration to remove undigested tissue chunks, the single cells are washed in Hanks Balanced Salt Solution (HBSS) prior to purification using single-step Ficoll gradient centrifugation. The purified cells are re-suspended in similar CM as the first method and dispensed in wells of 24-well plates at $1 \times 10^{6}$ cells $/ \mathrm{ml}$. The cultures are further incubated in a humidified incubator at $37{ }^{\circ} \mathrm{C}$ with $5 \% \mathrm{CO} 2$, and the monitoring step is done as the first method [8].

The last technique for TIL culture initiation is to use mechanical intervention such as homogenizer to disrupt cell aggregates. In the case of melanoma-derived TILs, physical disaggregation can be done using a disposable sterile mini homogenizer i.e. $50 \mu \mathrm{m}$ Medicon (C) chamber (Beckton Dickinson). In brief, several fragments, which are about $2 \mathrm{~mm}$ dimension each, are forced across a small grater inside the Medicon (C) chamber. The resulting cells and aggregates are then washed and re-suspended in CM without IL-2, followed by Ficoll gradient centrifugation. After 20 min' centrifugation at $2000 \mathrm{rpm}$, TIL containing lower interface is taken and washed. TILs are then plated in 24-well plates and cultured exactly as the first method [8].

\section{Rapid expansion protocol}

The term "rapid expansion protocol" (REP) was introduced by Rosenberg et al. [7]. It refers to a protocol for TIL expansion that is derived from lymphocytes after TIL extraction step. In this method, unselected lymphocytes are exposed to IL-2, which usually causes expansion to 40-50 millions of total cells within 2-4 w [12]. The resulting bulk of lymphocytes are called "young TILs", which then undergo either $\mathrm{CD}^{+}$enrichment step using magnetic-beads separation [13], or are directly subjected to activation step [12].

Subsequent activation step of either $\mathrm{CD}^{+}$-enriched or heterogeneous young TILs requires OKT3 (anti-CD3) antibody and 
high dose IL-2 in the presence of irradiated allogeneic feeder cell layer to induce TIL proliferation. Feeder cells are usually peripheral blood mononuclear cells (PBMCs) that are taken from healthy subjects with HLA mismatch, which help "young TILs" to further proliferate and be activated. Prior to co-culture step, PBMCs undergo irradiation (50 Gy) to inhibit their proliferation and induce apoptosis. In brief, PBMC $\left(2 \times 10^{8}\right)$ and "young TILs" $\left(1 \times 10^{6}\right)$ are cocultured in the presence of anti-OKT3 antibody in a medium that contains CM without IL-2 and AIM V medium at a ratio of $1: 1$. The cultures are grown in flasks and incubated at $37{ }^{\circ} \mathrm{C}$ with $5 \% \mathrm{CO}_{2}$. On day-2, IL-2 $(6000 \mathrm{IU} / \mathrm{ml})$ is added. Medium change is carried out by removing $50 \%$ of culture supernatant and replacing it with the same fresh $6000 \mathrm{IU} / \mathrm{ml}$ IL-2 containing medium. On day 6 and every day thereafter, cell concentration is determined and undergoes either continuing culture in additional flasks or being harvested for infusion purpose [8].
Prior to reinfusion, TILs should be prepared in an appropriate saline medium. The medium during REP is removed by centrifugation, and the cell pellets are washed in $0.9 \%$ sodium chloride. After another centrifugation step, TILs are then re-suspended in $0.9 \%$ sodium chloride with $2.5 \%$ human albumin and ready for intravenous administration [8].

\section{TIL manufacturing for solid tumour therapy}

Manufacturing TILs for cancer therapy requires a tight operational network for efficient clinical implementation. While tissue extraction and patient preparation toward the reinfusion process can be carried out in a clinic or hospital setting, TIL-manufacturing requires standard current good manufacturing practice (cGMP) process to meet a clinical-grade cellular product that contains highly activated T-cells before continuing to reinfusion step (fig. 2). An optimum culture system with efficient labour hour and manufacture cost is a priority in TIL manufacturing $[7,8,10,11]$.

\section{Harvest and preparation for infusion}

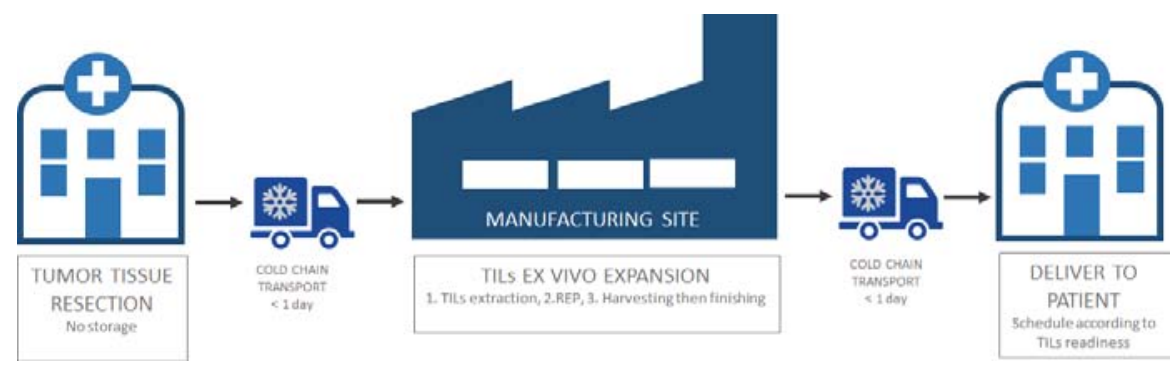

Fig. 2: A schematic diagram of TIL manufacturing for autologous therapy $[7,8,10,11]$

In general, the setting can be divided into three consecutive processes i.e.: (i) extraction of TILs from patient tumour tissue, (ii) T-cell manufacture that involves TIL extraction, rapid expansion and harvesting, and (iii) delivering TILs to lymphocyte-depleted patient. All processes should be carried out under a predetermined schedule according to manufacture capability $[7,8,10,11]$.

\section{Bioreactors}

Static tissue culture system was initially used in small-scale clinical trials on the basis of batch feeding. However, for larger-scale implementation, a static system is labour intensive, complicated and expensive. Therefore, simplifying the protocols is a must to meet cGMP compliant TILs production criteria. The major drawback of this static system is the need of manual intervention during culturing, which increases the production cost [14]. There are basically two types of bioreactors, which are designed to overcome this limitation, i.e. perfusion-based and gas-permeable bioreactors. These bioreactors have been described to simplify and streamline the production of autologous TIL therapy and can be integrated to REP workflow at the step of 'young TIL' ex vivo expansion [14-16]. In addition to the aforementioned bioreactors, a hollow fibre bioreactor is commonly used for $\mathrm{T}$ cell cultivation for cell therapy purposes. Commercially known as Quantum (C) Cell Expansion System, the hollow fibre bioreactor is basically a modification of perfusion-based bioreactor. Instead of using a bag as cell container, the system uses hollow-fibres with intra capillary surfaces to facilitate cell attachment. The yield of $\mathrm{CD}^{+} \mathrm{T}$ cells that were generated from this system was very promising [17]. Moreover, a hollow-fiber bioreactor has been implemented for TIL expansion with good results [18].

\section{Perfusion-based bioreactors}

Perfusion-based bioreactor was developed to overcome the limitation of static culture system that is solely dependent on manual intervention in the distribution of nutrients and oxygen, as well as the removal of waste products [19]. The system retains cells inside the bioreactor, while it is continually removing cell waste products and adding fresh media at the same rate. There are several ways to retain cells, such as by using capillary fibres (as developed by Quantum(C) $[17,18]$ or membranes to which the cells are attached to or another way that is based on a filtration system to keep the cells inside a chamber, and periodically exchange the media $[15,19]$. All types can be integrated with an automatic sensor that reports the culture microenvironment.

One common perfusion-based bioreactor that has been used for TILs expansion is GE WAVE bioreactor system (fig. 3). The system uses a pre-sterilized inflated cultivation chamber that is called a Cellbag during cultivation. It combines a rocking motion to generate a "wave" with a system that homogenously distributes oxygen and nutrients throughout the Cellbag, and a heater to provide the optimum temperature. Sensory patches are attached at the bottom of the Cellbag to measure the level of dissolved oxygen and medium $\mathrm{pH}[15,20-22]$.

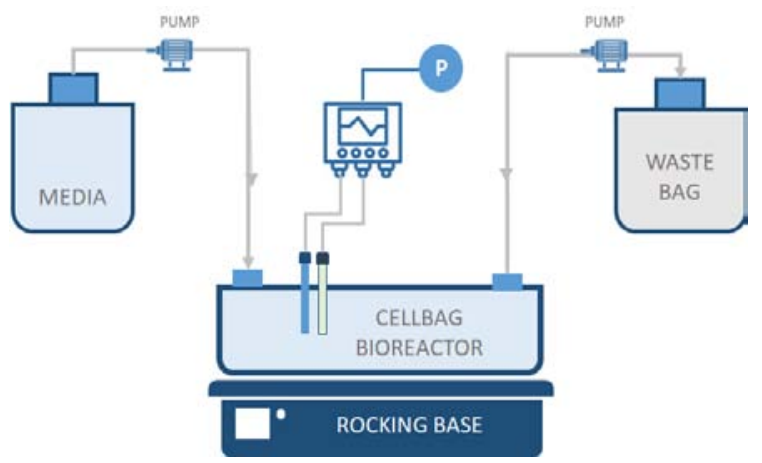

Fig. 3: A schematic diagram of a typical perfusion-based bioreactor [15, 20-22]]

This scalable system consists of a cell bag bioreactor, pumps to perfuse fresh medium while discharging waste at the same time, a range of optional controllers, including sensory patches to monitor $\mathrm{pH}$ and temperature, and a rocking base [15, 20-22]. 
In the setting of autologous TIL expansion, a bulk of 'young TILs' can be transferred into Cellbag Bioreactors and placed on a rocking base. The Cellbag is further rocked gently for rapid gas transfer and mixing. The perfusion functionality of the GE-WAVE allows automatic feeding and waste removal, thus reducing the labour intensive aspect for controlling cell microenvironment [15, 20-22]. Using this system, cells can be rapidly expanded to more than $10^{7}$ cells $/ \mathrm{ml}$ with a maximum capacity of up to 25L cell culture in a single bioreactor [15].

\section{Membrane bioreactors}

Basically, membrane bioreactor is a bioreactor that integrates a permeable-selective or semi-permeable membrane to allow growth of cells in suspension culture by providing active organic and/or gas exchange [16]. A typical membrane bioreactor, which was developed by Wilson Wolf Manufacturing, is named G-Rex flasks. It was designed to fulfil the requirement of highly expanded cells using larger medium volumes, particularly for TIL therapy platform. It consists of a common culture flask that is equipped with a silicone membrane, which is placed at the base of the flask; thus allowing efficient $\mathrm{O}_{2}$ and $\mathrm{Co}_{2}$ exchange (fig. 4) $[14,16]$. Unlike a perfusion bioreactor that requires mechanical assistance to provide a rocking motion, a gas-permeable bioreactor is able to support a cell culture with a large media volume in a static environment without the need of a mechanical agitation. Once the optimal seeding density has been reached, the nutrients are not a limiting factor, and cells will remain viable during longer period of culture $[14,16]$.

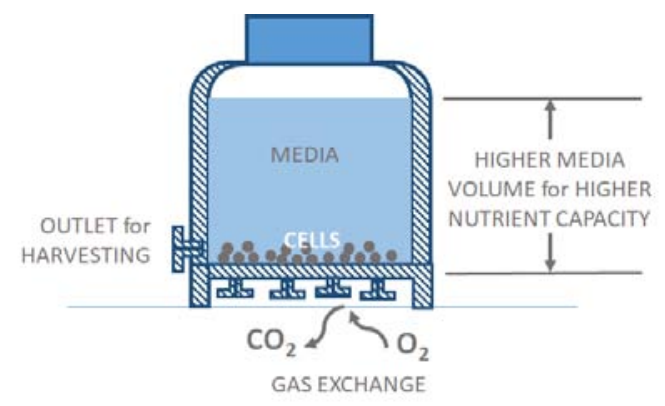

Fig.4: A cross-sectional view of a gas-permeable flask (membrane bioreactor) [16]

The flask has a gas-permeable silicone membrane at the bottom for efficient gas exchange, and should be placed inside a conventional incubator [16].

Using K562 cells, Bajgain et al. [16] showed that the lowest possible initial seeding density was $1.25 \times 10^{5}$ cells $/ \mathrm{cm}^{2}$, and $10 \mathrm{ml} / \mathrm{cm}^{2}$ of medium volume was enough to give a maximum cell number within 9-10 d. Therefore, implementation of $G$-Rex flasks into REP workflow might result in higher cell number, which was started from a minimum number of seeded cells, and a shorter culture period.

\section{Considerations in integrating bioreactor system into TIL manufacture}

All bioreactors described above offer substantial improvements over prior static system [16-22]. Several considerations should be taken into account regarding the scaling up of TIL manufacture. On the aspect of capital investment, the G-rex system $[14,16]$ offers a faster initiation for scaling up purposes, since it does not need additional specialized equipment. It offers a simple scaling up of flasks, which can be replaced by G-rex flasks $[14,16]$, according to patient's requirements that make the translation process from research laboratories to cGMP production and validation to become more convenient. However, the G-Rex system is not a completely closed system $[14,16]$. Integrating G-Rex flasks into REP process might be slightly complicated than the perfusion system (GE-WAVE or Quantum system) at the phase of cell harvesting and seeding [14].

On the other hand, the perfusion system offers a more convenient way on handling cultures in larger volumes, as the system facilitates medium exchange automatically without additional bioreactor bags for larger culture quantities [15, 20-22]. In comparison with the GRex system, which requires almost thirty G-Rex Flasks per patient treatment [14], the package of perfusion system, which can support up to $25 \mathrm{~L}$ culture in a single bioreactor [15], is more convenient.

GE-WAVE and G-Rex bioreactors were used in scaling up of TILs [14$16,20-22]$ and demonstrated impressive clinical results following TILs therapy for melanoma and cervical cancer [12-14], which further reinforce the commercial potential of TIL therapy. Further, the Quantum system $[17,18]$, which was used to culture TILs and scale-up CD3+T-cells, might also be a candidate for scaling up of TILs.

\section{CONCLUSION}

Adoptive TIL therapy holds potential for treating solid tumours in term of reducing adverse effects to host. Issues regarding limitation in cell number could be overcome by determining an optimum combination between cell source, culture initiation and bioreactor system. The WAVE and G-Rex bioreactors were successfully used in scaling up TILs in clinical settings. Both systems come with benefits and drawbacks to be considered. With an increasing number of commercially available advanced bioreactors including the Quantum system, facilitation of mass production of TILs in a short term culture period will become real soon.

\section{ACKNOWLEDGMENT}

This work was supported by a research grant from the Ministry of Research, Technology and Higher Education of the Republic of Indonesia, Hibah Penelitian Pengembangan 2019, contract no. NKB1804/UN2. R3.1/HKP.05.00/2019.

\section{FUNDING}

Nil

\section{AUTHORS CONTRIBUTIONS}

All the authors have contributed equally.

\section{CONFLICT OF INTERESTS}

The authors declare that they have no conflict of interest.

\section{REFERENCES}

1. Met O, Molgaard K, Chamberlain CA, Donia M, Svane IM. Principles of adoptive T cell therapy. Semin Immunopathol 2019;41:49-58.

2. Wang X, Riviere I. Clinical manufacturing of CAR T cells: the foundation of a promising therapy. Mol Ther Oncol 2016;3:16015.

3. Raghunathani K, Devi B. Chimeric antigen receptor for chronic lymphocytic leukemia-a review. Asian J Pharm Clin Res 2019;12:62-7.

4. Sulaiman AA, Al-Shamaa ZA, Al-Assadi ME. Evolving role of CAR Tcell in cancer immunotherapy. Int J Curr Pharm Res 2019;11:19-27.

5. Garber K. Pursuit of tumor-infltrating lymphocyte immunotherapy speeds up. Nat Biotechnol 2019;37:969-77.

6. Wickstrom S, Lovgren T. Expansion of tumor-infiltrating lymphocytes from melanoma tumors. Methods Mol Biol 2019;1913:105-18.

7. Rosenberg SA, Packard BS, Aebersold PM, Solomon D, Topalian SL, Toy SL, et al. Use of tumor-infiltrating lymphocytes and interleukin2 in the immunotherapy of patients with metastatic melanoma: a preliminary report. N Engl J Med 1988;319:1676-80.

8. Dudley ME, Wunderlich JR, Shelton TE, Even J, Steven A. Generation of tumour infiltrating lymphocytes cultures for use in adoptive transfer therapy for melanoma patients. J Immunother 2003;26:332-42.

9. Rohaan MW, Wilgenhof S, Haanen JBAG. Adoptive cellular therapies: the current landscape. Virchows Archiv 2019;474:449-61.

10. Dillman R, Schiltz P, DePriest C, Barth N, Beutel L, de Leon C, et al. Tumor-infiltrating lymphocytes and interleukin-2: dose and schedules of administration in the treatment of metastatic cancer. Cancer Biother Radiopharm 2004;19:730-7.

11. Geukes Foppen MH, Donia M, Svane IM, Haanen JBAG. Tumorinfiltrating lymphocytes for the treatment of metastatic cancer. Mol Oncol 2015;9:1918-35. 
12. Mayor P, Starbuck KS, Zsiros E. Adoptive cell transfer using autologous tumour infiltrating lymphocytes in gynecologic malignancies. Gynecol Oncol 2018;150:361-9.

13. Dudley ME, Rosenberg SA. CD8+enriched "young" tumour infiltrating lymphocytes can mediate the regression of metastatic melanoma. Clin Cancer Res 2010;16:6122-31.

14. Sommerville RP, Dudley ME. Bioreactors get personal. OncoImmunology 2012;1:1435-7.

15. Ready to process WAVE 25 bioreactor. Available from https://www.gelifesciences.com/en/sg/shop/cell-culture-andfermentation/rocking-bioreactors/systems/readytoprocesswave-25-rocker-p-05542 [Last accessed on 15 Jan 2020]

16. Bajgain P, Mucharla R, Wilson J, Welch, Anurathapan U, Liang $\mathrm{U}$, et al. Optimizing the production of suspension cells using the G-Rex "M" series. Mol Ther Methods Clin Dev 2014;1:14015.

17. Coeshott $C$, Vang B, Jones $M$, Nankervis B. Large scale expansion and characterization of $\mathrm{CD}^{+} \mathrm{T}$-cells in the quantum cell expansion system. J Transl Med 2019;17:258.
18. Malone CC, Schiltz PM, Mackintosh AD, Beutel LD, Heinemann FS, Dillman RO. Characterization of human tumor-infiltrating lymphocytes expanded in hollow-fiber bioreactors for immunotherapy of cancer. Cancer Biother Radiopharm 2001;16:381-90

19. Schmid J, Schwarz S, Staude RM, Sudhop S, Schaumann HC, Schieker M, et al. A perfusion bioreactor system for cell seeding and oxygen-controlled cultivation of three-dimensional cell cultures. Tissue Eng 2018;24:585-95.

20. Bausch M, Schultheiss C, Sieck JB. Recommendations for comparison of productivity between fed-batch and perfusion processes. Biotechnol J 2019;14:e1700721.

21. Somerville RP, Devillier L, Parkhurst MR, Rosenberg SA, Dudley ME. Clinical scale rapid expansion of lymphocytes for adoptive cell transfer therapy in the WAVE® bioreactor. J Transl Med 2012;10:69.

22. Donia M, Larsen SM, Met O, Svane IM. Simplified protocol for clinical-grade tumor-infiltrating lymphocyte manufacturing with use of the wave bioreactor. Cytotherapy 2014;16:1117-20. 